\title{
Terrorist incidents: strategic treatment objectives, tactical diagnostic procedures and the estimated need of blood and clotting products
}

\author{
Axel Franke ${ }^{1}\left(\mathbb{D} \cdot\right.$ Dan Bieler $^{1,2}\left(\right.$ D $\cdot$ Benedikt Friemert $^{3} \cdot$ Patrick Hoth $^{3} \cdot$ Hans-Christoph Pape $^{4}\left(\right.$ D $\cdot$ Gerhard Achatz $^{3}(\mathbb{D})$ \\ and the Deployment, Disaster and Tactical Surgery Working Group of the German Trauma Society
}

Received: 29 December 2019 / Accepted: 16 May 2020 / Published online: 16 July 2020

○) Springer-Verlag GmbH Germany, part of Springer Nature 2020

\begin{abstract}
Background Terrorism-related incidents that are associated with mass casualties (mass-casualty terrorist incidents) are a medical and organisational challenge for every hospital because of the special injury patterns involved, the time of the incident, the development of the situation, the initial lack of information, the number of injured, and the number of uninjured survivors who self-refer to a hospital.

Methods The Terror and Disaster Surgical Care (TDSC®) - Course was developed in order to address mass-casualty terrorist incidents and to provide surgeons with the specialist medical and surgical knowledge and skills required for these special situations. The focus of the TDSC ${ }^{\circledR}$ course is on how to provide surgical care and how to deploy scarce resources in a particular tactical situation in such a way that the number of survivors is maximised.

Results The effective management of such a tactical situation must be based on priorities and first and foremost requires the standardised sorting and categorisation of the injured at the hospital. The aim of triage, or the sorting of the injured, is to immediately identify patients with life-threatening injuries in environments with strained resources. The medical management of mass-casualty terrorist incidents requires tactical abbreviated surgical care (TASC) teams that have the skills needed to perform a primary survey and to provide care for casualties who need immediate surgery (triage category 1-T1). Initial fluid therapy should be restrictive (permissive hypotension) unless contraindicated. Clotting products are replaced in a standardised manner on the basis of patient requirements, which are calculated using rapidly available surrogates (blood gas analysis). Blood products can be administered or kept available depending on risks and triage categories. The highest priority should be given to the identification and management of haemodynamically unstable patients who require immediate surgery for injuries associated with bleeding into body cavities $(\mathrm{T} 1++)$.

Conclusion The recommendations and approaches described here should be considered as proposals for hospitals to develop standards or modify well-established standards that enable them to prepare themselves successfully for situations (e.g. masscasualty terrorist or shooter incidents) in which their resources are temporarily overwhelmed.
\end{abstract}

Keywords Terrorist incidents $\cdot$ Blood products $\cdot$ TDSC $\cdot$ Tactical care $\cdot$ Triage $\cdot$ Tactical diagnostic procedures

Axel Franke and Dan Bieler contributed equally to the paper.

Dan Bieler

dr.dan.bieler@t-online.de

1 Department for Trauma Surgery and Orthopaedics, Reconstructive and Hand Surgery, Burn Medicine, German Armed Forces Central Hospital Koblenz, Rübenacher Straße 170, 56072 Koblenz, Germany

2 Department of Orthopaedics and Trauma Surgery, Heinrich Heine University Hospital Düsseldorf, Moorenstraße 5, 40225 Düsseldorf, Germany
3 Department for Trauma Surgery and Orthopaedics, Reconstructive and Septic Surgery, Sportstraumatology, German Armed Forces Hospital Ulm, Oberer Eselsberg 40, 89081 Ulm, Germany

4 Department of Trauma, University Hospital Zurich, Raemistrasse 100, 8091 Zurich, Switzerland 


\section{Introduction}

Terrorism-related and shooter incidents present enormous challenges not only for emergency service responders at the scene but also for nearby hospitals, which may be confronted with unusual patterns of injury and large numbers of patients [1-3].

Depending on the type of incident, these situations can cause mass casualties (e.g. mass-casualty terrorist incidents) and overwhelm the treatment resources of a hospital $[4,5]$.

Especially perforating and penetrating injuries to body cavities or junctional injuries (in the cervical, axillary or inguinal regions) from projectiles, projectile fragments or other fragments lead to an increased number of patients with life-threatening bleeding that requires surgical management $[6,7]$.

These special injury patterns and a disproportionate number of haemodynamically unstable casualties who require rapid intervention contribute to the dynamic nature of terrorist incidents, which further exacerbates the situation in terms of both the quantity and quality of care $[4,7,8]$.

Terrorist incidents may present not only as war-like scenarios with the injury patterns described above but also, for example, as traffic accidents, as the recent attacks at Breitscheidplatz in Berlin in 2016 and on the Promenade des Anglais in Nice in 2016 have shown [9-11].

Maximising the number of survivors requires an initial tailored tactical response to the exceptional situation of a mass-casualty terrorist or shooter incident, the identification of treatment priorities, and the optimum use of available resources.

In such a situation, it must be accepted that the availability of only limited resources is the basis and justification for triage or the sorting of casualties into priorities until reliable information on the situation suggests otherwise. Accurate triage is then the basis for the appropriate allocation of diagnostic and treatment resources according to priorities.

These principles make clear that hospitals must inevitably modify the further management of patients and the organisation of care $[4,8,10]$.

The objective of this article is to assess how team organisation, diagnostic procedures and treatment can be structured and modified on the basis of Terror and Disaster Surgical Care (TDSC $®)$ principles in such a way that a maximum number of patients can receive optimum care.

\section{Terror and Disaster Surgical Care (TDSC ${ }^{\circledR}$ )- background and principles}

\section{$\operatorname{TDSC}^{\circledast}$ and tactical care}

Following a description of the background and special aspects of terrorism-related incidents, their consequences for hospitals, and the associated development of the TDSC $®$ course (see article 1 of this Focus-On - Issue; Friemert et al.), this article explains how the focused deployment of diagnostic, interventional, surgical and other resources can contribute to maximising the number of survivors through the provision of what may be termed minimum essential care for as many patients as possible (Fig. 1).

Unlike in the setting of the individualised management of patients in the trauma room (e.g. advanced trauma life support-ATLS ${ }^{\circledR}$ by trauma teams) and the surgical management of patients on the basis of the principles of early total care (ETC) and damage control surgery (DCS), major incidents may require the allocation of resources in accordance with tactical abbreviated surgical care (TASC) principles until capacities are no longer overwhelmed [12].

Whereas the damage control (DC) approach is based on an overall assessment of the individual patient, TASC depends on the situation, which determines the type of approach and the extent of initial diagnostic and medical or surgical procedures required to ensure immediate survival.

\section{$\operatorname{TDSC}^{\oplus}$ and the role of triage}

Effective in-hospital management of patients must be based on priorities and necessitates the standardised sorting and categorisation of the injured at the hospital. This is the task of the senior triage coordinator.

The aim of triage, or the sorting of the injured, is to identify casualties with life-threatening injuries in environments with scarce resources. Triage category 1 (T1) (red) encompasses patients with an acute lifethreatening condition. T2 (yellow) patients require urgent treatment. If they do not receive immediate care, they may suffer a loss of function but the underlying injury is not an immediate life-threatening condition.

T3 (green) patients are able to walk and present with minor injuries that may require monitoring. They must be examined by a physician as soon as sufficient resources are available.

Mass-casualty terrorist incidents are associated with a large number of penetrating injuries. Haemodynamically unstable patients with major bleeding into body cavities are categorised as $\mathrm{T} 1++$ (red). 


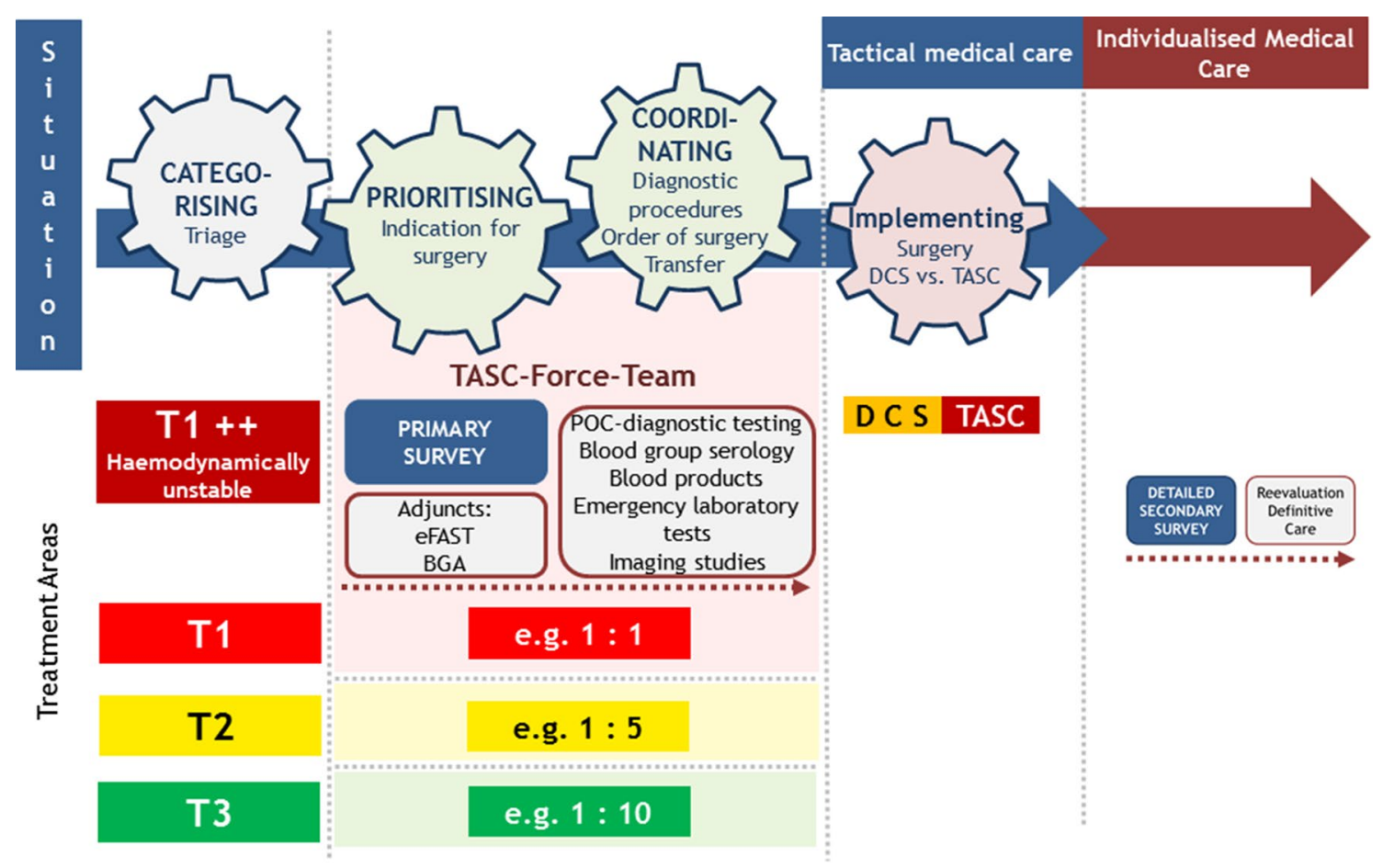

Triage area

Treatment team

Fig. 1 POC point of care, DCS damage control surgery, TASC tactical abbreviated surgical care, eFAST extended focused assessment with sonography in trauma, $B G A$ blood gas analysis

These patients can only be stabilised and can only survive if they receive rapid surgical treatment. In masscasualty terrorist incidents, surgical care for these patients can only be achieved if organisational aspects and the allocation of resources are optimised.

\section{Modifying medical care on the basis of tactical aspects}

What, then, are the options for modifying medical care on the basis of tactical aspects in the setting of a terrorismrelated incident in which resources are limited?

1. Modification of treatment aims Eminence-based treatment aim (survival of as many victims as possible) instead of evidence-based individualised approaches (ETC and DCS—best individual outcomes).

2. Focused allocation of treatment resources Surgical care for patients who can be treated surgically with available technical equipment and who have the best prognosis in terms of survival and full recovery (e.g. neurological function). Transfer of haemodynamically stable patients with injuries that cannot be managed surgically with available resources (e.g. head or vascular injuries).
3. Formation of treatment teams and operating teams Formation of treatment teams (TASC teams) that have the skills needed to ensure patient survival (for example at a treatment station) instead of interdisciplinary teams that provide optimum individualised trauma room care based on injury patterns Formation of teams with experience in the management of injuries involving body cavities and initial surgery focusing on bleeding control.

4. Temporary acceptance of compromised conditions in patients Permissive hypotension. Permissive anaemia

5. Focused diagnostic laboratory and imaging procedures Bedside and point-of-care laboratory tests required for diagnosis and successful surgical management. Initial focus on routine laboratory tests for ensuring the availability of patient-specific blood products as early as possible. Diagnostic laboratory tests only if relevant for treatment.

6. Calculated instead of targeted replacement of blood and clotting products Replacement of oxygen carriers and clotting products on the basis of the estimated blood loss as determined using surrogates (e.g. haemoglobin, base excess, thrombin time, international normalised ratio), severity of shock, and trauma-induced coagulopathy (TIC). 
Whereas items 1 to 3 are the subjects of other articles of this special issue, items 4 to 6 are addressed in detail here.

\section{TDSC $^{\circledR}$ - organisational elements in hospitals}

In-hospital triage is essential since it provides an important basis of decisions regarding the prioritisation of available resources in a situation in which normal capacities are overwhelmed and untriaged patients are likely to arrive at a hospital in an uncoordinated manner.

After the sorting of patients by the senior triage coordinator (CATEGORISING), TASC teams perform the primary survey and the emergency operational and medical coordinator (EOMC, ZONK) prioritises patients for further diagnostic and surgical procedures (PRIORITISING).

Haemodynamically unstable patients who require immediate surgery for stabilisation are directly identified. The ZONK arranges only for diagnostic and other procedures that are essential for ensuring patient survival and determines the scope of the measures to be implemented (COORDINATING). After the type and scope of surgery are defined, patients are taken to the operating room and undergo surgery as planned (IMPLEMENTING).

Under these exceptional conditions, the initial focus is on maximising the number of survivors and not on achieving the best possible functional outcome for every patient. The management of mass-casualty events requires rapid, targeted and effective decision-making based on simple criteria, robust leadership and direct communication.

The primary purpose of all measures is to rapidly identify haemodynamically unstable patients with life-threatening uncontrollable bleeding $(\mathrm{T} 1++)$, to immediately arrange for and perform surgery without delay, and to make available essential resources.

\section{The role of triage in the focused allocation of resources-categorising}

The rapid provision of care designed to ensure patient survival decisively depends on the in-hospital sorting of patients by the senior triage coordinator on the basis of hospital-specific aspects of how to categorise patients, how to assign patients to appropriate treatment areas, and thus how to determine further diagnostic studies and the further management of patients. Both under-triage and over-triage have been shown to adversely affect patient survival and the allocation of resources [13].

Appropriate triage is a prerequisite for the further management of patients in treatment areas on the basis of priorities and triage categories [14-16].

After rapid triage at the hospital, extended focused assessment with sonography in trauma (eFAST) and the primary survey by a TASC team in an appropriate hospital treatment area allow overall injury severity to be assessed and enable the emergency operational and medical coordinator (EOMC) to sort patients and especially haemodynamically unstable patients into priorities for further treatment [12].

\section{Formation of treatment teams and operating teams}

\section{TASC teams with appropriate skills instead of interdisciplinary trauma room teams}

The advanced trauma life support (ATLS $®$ ) system and the current version of the German Level 3 Guideline on the Treatment of Patients with Severe/Multiple Injuries [17] address the management of patients in a fully equipped trauma room and define team composition, diagnostic procedures and necessary resources accordingly.

Patients should receive the best possible care and individualised treatment that is tailored to the individual patient, the mechanisms of injury, and the related suspected injury patterns. In a situation in which resources are overwhelmed, the preparation and organisation of care must be modified in accordance with the above-mentioned principles of TDSC $®$.

Redundant skills and alternative treatment options are absolute exceptions. In a mass-casualty terrorist event or another major incident, the injured are triaged at the hospital to the treatment area that is most appropriate to their specific triage category.

This is where an ATLS ${ }^{\circledR}$ primary survey is conducted and surgeons with the required skills initiate treatment designed to ensure the survival of the patient. The skills that these surgeons must have in order to conduct a primary survey in accordance with ATLS $®$ include the induction of anaesthesia and intubation, surgical airway, needle decompression of the chest, chest drain insertion, pericardiocentesis and eFAST, pressure dressings, tamponade, tourniquets, and the application of a pelvic binder.

Small effective teams (TASC teams) should be formed. Together the members of TASC teams must master the aforementioned skills and be able to use them in tactical settings.

Depending on the assessment of the overall condition of the patients and the development of the situation, the emergency operational and medical coordinator (EOMC) determines priorities for surgical procedures, the type and scope of surgical and diagnostic procedures, the planned 
management of patients, and the order in which patients should undergo surgery.

\section{Minimum essential care instead of best possible care}

Apart from basic supplies and equipment (e.g. suction unit, ventilator, ultrasound device, monitors), this approach requires only a core team (TASC team) that has the necessary skills and may consist of a specialist in anaesthesiology and a resident in surgery - or a specialist in surgery and a resident in anaesthesiology - and an emergency department (ED) nurse. Teams are formed on the basis of skill levels (e.g. ATLS® providers) and clinical experience and medical or surgical specialties.

Unlike the "best possible care" principle, this approach does not require a trauma room and an interdisciplinary team of specialists who act as one but a treatment team (TASC team) that provides patient-focused treatment in the hospital. These personnel and equipment resources provide the basis for ensuring the delivery of minimum essential care wherever required and thus for ensuring the survival of patients.

Triage category 1 (T1) (red) casualties by definition present with immediate life-threatening conditions. Accordingly, one TASC team is required per T1 (red) patient.

Depending on the availability of personnel and other resources, this requirement of one treatment team per $\mathrm{T} 1$ patient can be met if initial care for T2 (green) casualties is provided at a ratio of, for example, one TASC team to five patients and for T3 (green) patients at a ratio of 1 to 10.

\section{Formation of TASC teams and operating teams}

The successful surgical management of casualties requires that a senior triage coordinator and an emergency operational and medical coordinator (EOMC) are designated and organise the provision of care and that appropriate personnel are deployed to the various treatment areas (e.g. RED, operating room, YELLOW, GREEN, laboratory, X-rays, intermediate care, intensive care) in a rapid and focused manner. In addition, successful surgical care requires that treatment teams (TASC teams) and operating teams be composed in such a way that the required skills are available.

Whereas the availability of a sufficient number of specialists with TASC team skills (e.g. ATLS ${ }^{\circledR}$ providers) can be ensured as part of the preparation for major incidents, the formation of operating teams and the provision of appropriate amounts of sterile supplies, surgical instrument sets and, if required, disposable items are an organisational challenge.
The formation of teams with experience in the management of injuries involving body cavities is particularly difficult in the initial situation when resources are temporarily overwhelmed. A skills matrix may be created in order to organise the composition of teams and assess training needs as part of the preparation for major incidents.

First the required skills are defined. Then the persons responsible for continuing training (hospital directors or department heads) and surgeons are asked about these skills with a view to assessing what skills surgeons master, what skills they are comfortable with, and what skills they themselves and their superiors believe they can perform in an emergency situation.

This approach may give evidence of an imbalance between requirements and available capabilities which must be closed, for example through continuing training, courses and/or organisational measures.

Another possible way to address the lack of specialists in a major incident is to strengthen operating teams by including other specialist surgeons from within the hospital (e.g. vascular or visceral surgeons) or other personnel from outside the hospital (e.g. surgeons and scrub nurses) for special surgical procedures.

\section{Temporary acceptance of compromised conditions in patients}

Bleeding patients whose circulation is centralised in shock inevitably experience hypotension. The concept of permissive hypotension is based on the idea that the temporary acceptance of a lower-than-normal blood pressure reduces the total loss of blood caused by the underlying injury.

Unless contraindicated (major traumatic brain injury), restrictive fluid therapy is used and no measures are taken to raise mean arterial pressure (e.g. administration of vasopressors, patient positioning) until bleeding is controlled $[18,19]$.

Studies have demonstrated that this approach led to a decrease in total blood loss and transfusion requirements in patients with penetrating injuries and bleeding into body cavities. In addition, they suggested that overall mortality and the incidence of organ dysfunction can thus be reduced over time. Permissive hypotension, however, is controversial since it involves a deliberate extension of the period of circulatory compromise and the state of shock in a patient who is in poor condition. For this reason, this strategy should be used only in tactical medical situations that require exceptional measures [20,21].

The same applies to the acceptance of lower haemoglobin $(\mathrm{Hb})$ levels in situations where resources are limited. 
According to the current version of the German Trauma Society (DGU®) Level 3 Guideline on the Treatment of Patients with Severe/Multiple Injuries, a target $\mathrm{Hb}$ level of $7-9 \mathrm{~g} / \mathrm{dl}$ is safe in patients with severe/multiple injuries [17].

Case reports, studies involving special patient populations, and a current report of a consensus conference suggest, however, that even lower Hb levels $(<7 \mathrm{~g} / \mathrm{dl})$ may be temporarily accepted if blood rheology is optimised [22]. This tactical medical approach, which may be used when resources are overwhelmed in a mass-casualty terrorist incident, is known as permissive anaemia.

Decisions regarding temporarily acceptable thresholds, the allocation of resources (blood products) to patients, and targets for these resources depend on the risk assessment by a healthcare provider (EOMC) and on the evaluation of comorbidities and confounders (e.g. age, gender, ischaemic heart disease, overall probability of survival).

\section{Focused diagnostic laboratory and imaging procedures}

\section{Diagnostic laboratory tests}

An assessment of the severity of haemorrhagic shock, trauma-induced coagulopathy (TIC) and transfusion requirements is of decisive importance for initial stabilisation (resource planning), the success of subsequent surgical management and overall mortality. This also applies to situations in which resources are temporarily overwhelmed.
A number of studies have demonstrated that, for example, the severity of shock estimated on the basis of blood pressure, $\mathrm{pH}$ and base excess correlated with transfusion requirements [23-25]. These values can be obtained from standard blood gas analyses (BGA) that can be performed as bedside or point-of-care (POC) diagnostic tests [26].

There is also evidence suggesting that the Hb level on admission is a surrogate for estimated blood loss and provides the basis for a successful calculation of requirements for the replacement of clotting products [27-29]. The international normalised ratio (INR) is discussed as another parameter for an assessment of trauma-induced dilutional coagulopathy and has been established as a POC test for patients on anticoagulant therapy.

At the same time, rotational thromboelastometry (ROTEM) and FIBTEM are used in a standardised manner in many medical centres as bedside or POC tests for assessing coagulation and guiding replacement therapy [30-32].

These diagnostic tests are available more rapidly than routine emergency diagnostic procedures but they too require personnel and material resources as well as time and expertise and thus training.

Although permissive hypotension and a low total $\mathrm{Hb}$ level (permissive anaemia) in young patients without known preexisting or co-existing conditions can be initially accepted (unless contraindicated), the administration of blood products during the course of treatment is essential for the management of haemorrhagic shock and the prevention of organ dysfunctions and complications [33, 34].
Fig. 2 POC point of care, $B G A$ blood gas analysis, $H b$ haemoglobin, INR international normalised ratio, $T T$ thrombin time, $a P T T$ activated partial thromboplastin time

\section{TerrorMASCAL: Focused use of diagnostic procedures}

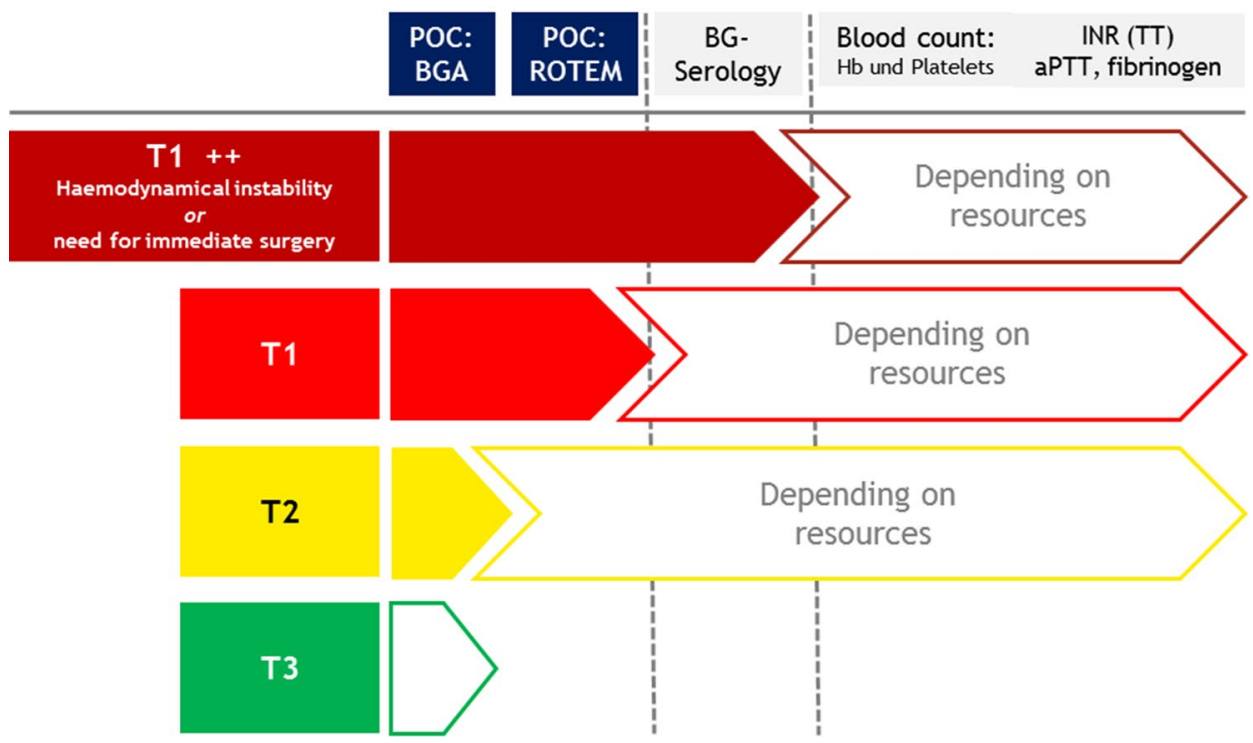


A period of limited resources initially requires the focused allocation of diagnostic tools and blood products to patients with haemodynamical instability secondary to bleeding ( $\mathrm{T} 1++-$ haemodynamic instability or need for immediate surgery) and with a high probability of requiring immediate surgery for controlling haemorrhage and ensuring survival.

In a mass-casualty terrorist incident, the objective is to reduce routine laboratory tests in order to save resources, for example for the use of blood products and serology blood tests since supplies such as universal donor blood (type $\mathrm{O}$ and $\mathrm{Rh}$ negative blood) are likely to become scarce soon.

Figure 2 shows a proposal for the focused use of diagnostic laboratory tests in accordance with triage categories. This approach allows diagnostic procedures to be deployed in such a way that time and personnel can be saved in a risk-adapted manner.

The purpose of blood gas analyses (BGA) in T2 patients, for example, is to detect occult blood loss. Depending on the availability of resources, BGA may be performed in T3 patients in order to detect respiratory failure in clinically suspected patients and to exclude blast injury to the lung.

\section{Diagnostic imaging}

In a situation of scarce resources, the time required to perform a diagnostic imaging test must always be weighed against a possible delay in carrying out surgery and against the associated requirements in terms of personnel, material and equipment resources that are urgently needed in the management of other patients.

When the initial focus of attention is on haemodynamically unstable patients with penetrating injuries to body cavities, extended focused assessment with sonography in trauma (eFAST) is a rapid and noninvasive method for identifying the body cavity affected by bleeding. Conditions with similar signs and symptoms such as pericardial tamponade and tension pneumothorax can be ruled out [35-37]. The need for surgery can thus be confirmed using eFAST.

Initially, further diagnostic studies such as chest X-rays, pelvic X-rays or a trauma scan with contrast enhancement are relevant for treatment only if they provide additional information that helps improve outcome or if eFAST results are not conclusive.

It would be interesting to discuss whether an increased number of negative exploratory laparotomies or thoracotomies as a result of wide indications for surgery are tactically acceptable in a situation of limited resources. This should be prevented by targeted diagnostic procedures since surgical capacities are absolutely scarce in the initial period of a situation of limited resources.

The advantages of contrast-enhanced whole-body computed tomography (CT) scans (trauma scans) are undisputed when individualised medical care can be provided to trauma patients $[38,39]$. In a mass-casualty terrorist incident in which resources are temporarily overwhelmed, the emergency operational and medical
Fig. $3 \mathrm{Hb}$ haemoglobin, ETC early total care, $D C S$ damage control surgery, TASC tactical abbreviated surgical care

\section{TerrorMASCAL: Calculated therapy}

- Define an "algorithm for coagulation management"

- Establish a damage control resuscitation protocol

- Replace clotting products for the management of trauma-induced coagulopathy on the basis of $\mathrm{Hb}$

- Further initial therapy calculated on the basis of blood gas analysis results

- Permissive hypotension and permissive anaemia

\section{Individualised medical care}

Aim: Survival, function, optimum reconstruction, full recovery

ETC
Routine
diagnostic procedures
Targeted
Individualised
replacement therapy

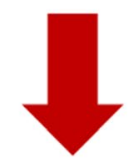

TASC

Focused emergency diagnostic procedures

Calculated, then delayed individualised replacement therapy 
coordinator (EOMC) must, however, carefully decide how to allocate this diagnostic resource among the many patients.

A CT scan always requires a considerable amount of resources since patients must be transported to the scanner, may need to be moved, and may require additional intravenous access and changes of position. Waldeck et al. reported that time can be saved when an abbreviated standardised algorithm is established which focuses on the detection of bleeding and life-threatening conditions and which can be useful to identify typical terrorism-related injuries in a mass-casualty terrorist incident [40].

\section{Calculated instead of targeted replacement of blood and clotting products}

Based on the same principles as above, the objectives and indications for the replacement of clotting products (Fig. 3) too can be adapted to triage categories and priorities for immediate surgery.

As described above, the temporary acceptance of permissive hypotension and permissive anaemia can lead to tissue hypoxia and prolong or worsen the shock state. In addition, it can cause acidosis and thus aggravate coagulation dysfunction (the triad of death).

Whereas requirements for the replacement of clotting products can be calculated, the assessment of a prolonged state of shock is based on a clinical evaluation of the patient and laboratory findings. The $\mathrm{pH}$ value, measured by blood gas analysis, can be temporarily influenced, for example by modifying ventilation or administering buffers.

Local coagulation is of prime importance for the success of measures taken by a surgeon to control bleeding into a body cavity or from an injury to a parenchymatous organ.

The main purpose of all efforts is to provide for optimum coagulation conditions at the time of surgical intervention. If the patient's general condition allows for permissive hypotension and permissive anaemia, the replacement of fluids and oxygen carriers should be started in a TASC setting only after bleeding is controlled.

Patients requiring surgery (all $\mathrm{T} 1++$ patients and selected $\mathrm{T} 1$ patients) should receive calculated amounts of tranexamic acid, fibrinogen and prothrombin complex concentrates (PCC) as soon as possible in accordance with the current European guideline [26].

Hospitals must decide on an individual basis how these recommendations can be implemented (e.g. massive transfusion protocols) in order to ensure preparedness for a mass-casualty terrorist or other major incident.

The administration of blood products (packed red blood cells, fresh frozen plasma and platelets), however, should not be based on automatic indications used in the setting of individualised medical care since these blood products are scarce resources during the initial stage of a mass-casualty terrorist incident. Supplies of blood products are limited and considerable routine laboratory time and personnel resources are required before patient-specific cross-matched red blood cells are released.

\section{Theoretical need for blood products in a mass-casualty terrorist incident}

The German Trauma Society (DGU®) Guideline on the Treatment of Patients with Severe/Multiple Injuries recommends that six units of $\mathrm{O} \mathrm{Rh}$ negative packed red blood cells (PRBCs) be stockpiled for every patient who meets the activation criteria for the trauma team (e.g. presence of a penetrating injury) $[17,41]$.

In 2016, Bieler et al. conducted a survey based on data from the trauma registry of the German Trauma Society (TraumaRegister DGUß) and reported that $26.9 \%$ of patients with penetrating gunshot wounds and $18.4 \%$ of patients with penetrating stab wounds required transfusions within the first $24 \mathrm{~h}$ in the setting of individualised medical care. Approximately $6.7 \%$ of these patients required massive transfusions, i.e. more than 10 units of PRBCs [42].

By contrast, Geissler et al. reported in 2015 in a subgroup analysis that patients with multiple trauma on average required 8-10 units of PRBCs, 17 units of fresh frozen plasma (FFP), and 3 units of platelets during their hospital stay [43].

In 2012, Bala et al. analysed data from 137 patients who had been admitted to an Israeli Level I trauma centre following a suicide bombing attack. Bala et al. reported that almost $40 \%$ of these patients, most of whom had sustained blast injuries, required blood transfusions during the first two hours. The mean number of transfused PRBCs was 4 (range 0-59) units per patient. It is interesting to note that the number of ordered PRBC units was twice as high as the number of transfused PRBC units. This result clearly demonstrates the adverse consequences of overtriage and large-scale orders of PRBCs. Only $10 \%$ of the patients required massive transfusions [44].

A retrospective analysis of transfusion requirements following the attacks in Paris and Nice revealed that patients who required blood transfusions received a mean number of 4-8 RBC units in Paris and 3-7 RBC units in Nice as well as a mean number of 4 units of FFP and 0-4 units of platelets in both Paris and Nice [45]. Whether the percentage of patients receiving blood products, nor the range of the overall need were shown in detail.

Where possible, women received type $\mathrm{O} \mathrm{Rh}$ negative RBCs. Men received either $\mathrm{O} \mathrm{Rh}$ positive or negative 
Table 1 Blood supplies in a trauma network (TraumaNetzwerk® DGU®)

Blood supplies in a trauma network (TraumaNetzwerk ${ }^{\circledR}$ DGU $^{\otimes}$ ) (telephone survey, weekdays at 10:30 a.m.)

\begin{tabular}{c|c|c|c|c|c|c|c}
$\begin{array}{c}\text { PRBC } \\
\text { units }\end{array}$ & STC & STC & STC & RTC & RTC & RTC & LTC \\
\hline 0 & 48 & 35 & 26 & 28 & 37 & 49 & 26 \\
\hline A & 61 & 38 & 32 & 20 & 31 & 42 & 32 \\
\hline B & 16 & 0 & 9 & 3 & 2 & 10 & 9 \\
\hline AB & 0 & 0 & 6 & 3 & 0 & 3 & 6 \\
\hline & 125 & 54 & 73 & 73 & 70 & 101 & 73 \\
\hline $\begin{array}{c}\text { FFP } \\
\text { units }\end{array}$ & STC & STC & STC & RTC & RTC & RTC & LTC \\
\hline 0 & 30 & --- & 9 & --- & 6 & 29 & 0 \\
\hline A & 30 & --- & 5 & --- & 10 & 32 & 2 \\
\hline B & 15 & --- & 11 & --- & --- & 24 & 0 \\
\hline AB & 40 & --- & 10 & --- & 10 & 22 & 4 \\
\hline-115 & 114 & 35 & 20 & 26 & 107 & 6 \\
\hline
\end{tabular}

$P R B C$ packed red blood cell, STC supra-regional trauma centre, $R T C$ regional trauma centre, $L T C$ local trauma centre, FFP fresh frozen plasma
RBCs. As soon as blood typing results were available, all patients received patient-specific compatible RBCs.

The aforementioned studies suggest that every haemodynamically unstable $\mathrm{T} 1++$ patient requires a mean of 6 PRBC units, 4 FFP units and 2 platelet units. The question now arises whether these transfusion requirements can be met by current hospital blood supplies.

In 2018, we conducted a telephone survey (on weekdays at 10:30 a.m.) that involved a medium-sized trauma network (DGU® TraumaNetzwerk ${ }^{\circledR}$ ). We assessed the blood supplies stored at local trauma centres (LTCs), regional trauma centres (RTCs) and supra-regional trauma centres (STCs) (Table 1).

Assuming that every haemodynamically unstable $\mathrm{T} 1++$ patient requires 6 PRBC units, 4 FFP units and $0-4$ platelet units in a mass-casualty terrorist incident, Table 1 gives an idea of how the transfusion requirements of patients can, or must be, managed using available supplies. If possible, $50 \%$ of the transfusion requirements should be met with universal donor blood (type $\mathrm{O}$ and $\mathrm{Rh}$ negative and positive PRBCs).

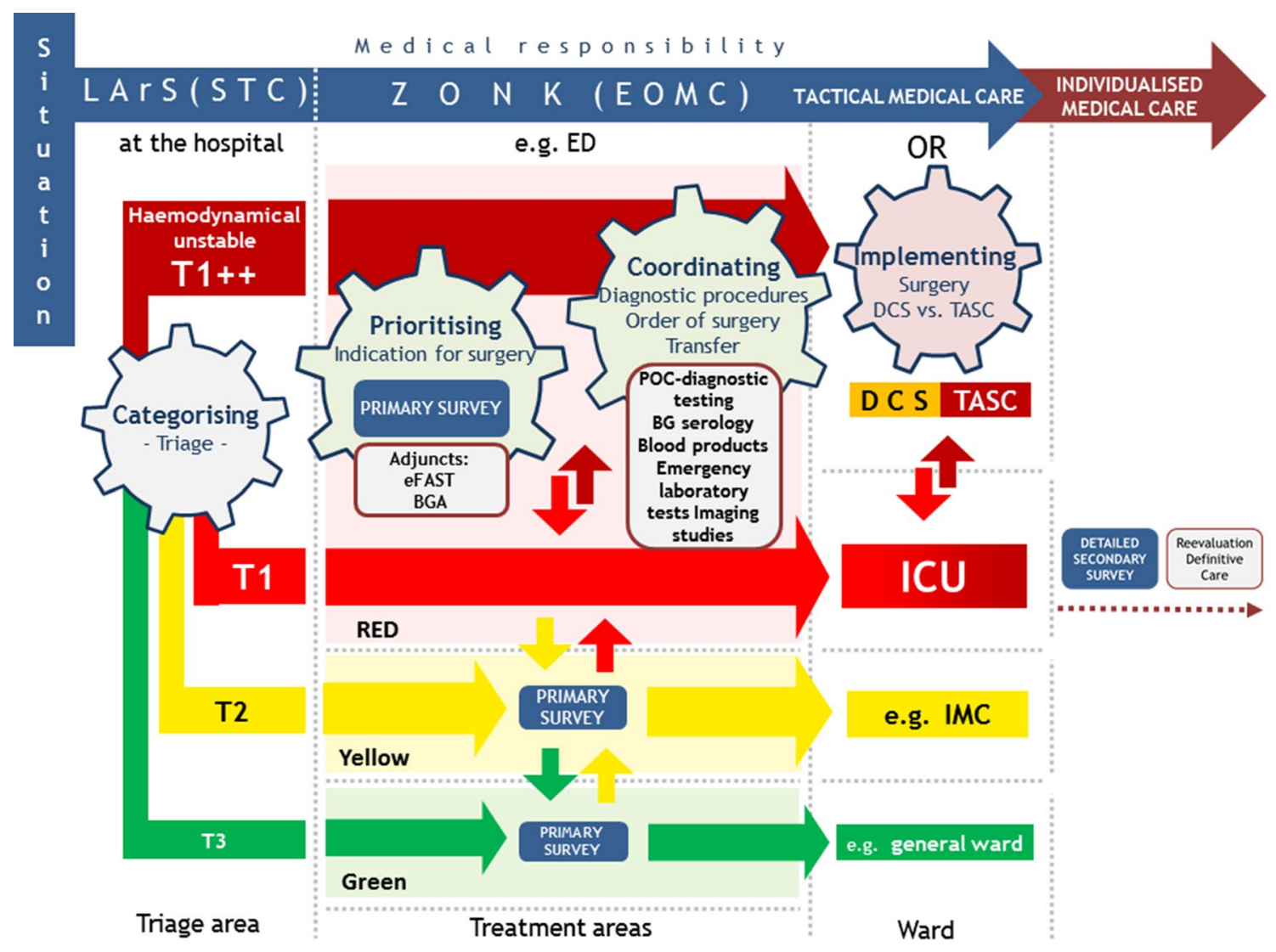

Fig. $4 \mathrm{LArS} / \mathrm{STC}$ senior triage coordinator, ZONK/EOMC emergency operational and medical coordinator, $E D$ emergency department, $I C U$ intensive care unit, IMC intermediate care, $P O C$ point of care,
$O R$ operating room, DCS damage control surgery, TASC tactical abbreviated surgical care 


\section{Case example: penetrating abdominal injury}

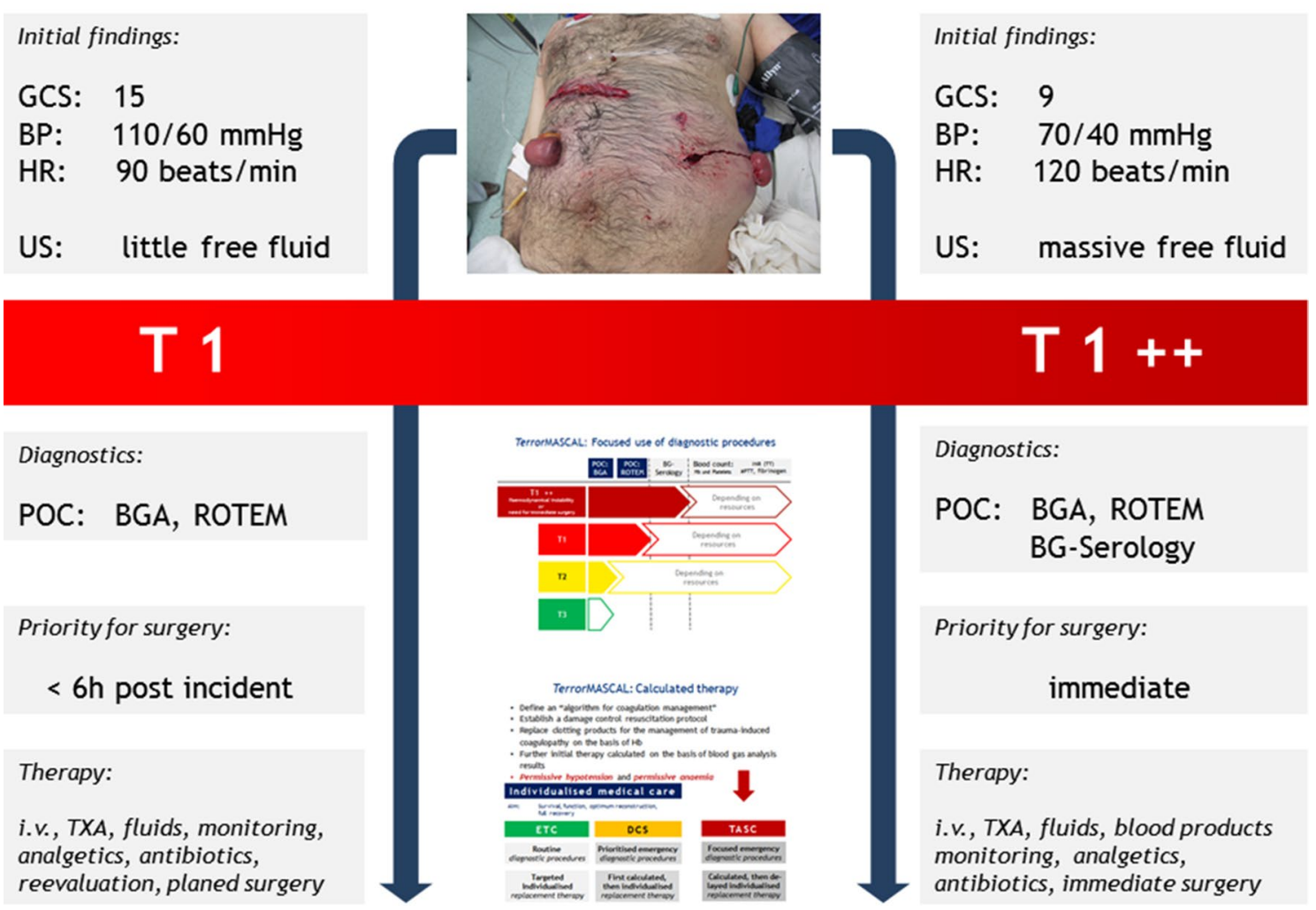

Fig. 5 Case example: penetrating abdominal injury in a mass casualty situation

\section{Patient flow in environments with strained resources}

Figure 4 gives an example of how to manage in-hospital patient flow and care on the basis of the results of initial triage on admission, the organisation of immediate lifesaving measures as advised by the ATLS $®$ system, and the tactical and strategic principles of TDSC®.

Following categorisation, T1 (RED) patients must rapidly have a primary survey conducted by a TASC team, which has the skills required to provide this type of care.

Further diagnostic procedures are then performed depending on triage categories, the urgency for surgical management, and the overall situation.

Figure 5 shows a case example with a patient showing up with a penetrating abdominal injury in a mass casualty situation. This case demonstrates that individual decision making in the group of red categorized patients is not that easy and point out the crucial role of the emergency operational and medical coordinator (EOMC). The EOMC determines the further management of patients, the priorities for diagnostic and/or surgical procedures, and the type and scope of diagnostic (laboratory and imaging) procedures that are essential for ensuring patient survival and the success of surgical stabilisation (COORDINATING).

Laboratory tests can, for example, be performed as focused POC diagnostic procedures (Fig. 4). All required measures are then implemented by the TASC team or an operating team as rapidly as possible.

Once stabilised, patients are transferred from the operating room to, for example, the intensive care unit (ICU). When patients develop haemodynamical instability on the ICU despite all medical and surgical measures, the EOMC must again determine priorities for further diagnostic and surgical procedures and must make necessary arrangements.

T2 (YELLOW) patients are monitored in appropriate treatment areas where they are managed by treatment teams. Depending on the availability of capacities, they are transferred to (RED) treatment areas and have a primary survey that is conducted by treatment teams, for example following the management of $\mathrm{T} 1$ patients.

When a (T2 or T3) patient develops a life-threatening injury-related condition or when a surgeon assigns a 
patient to a higher triage category, the patient can rapidly have a primary survey by an appropriate treatment team or can be transferred to the next higher treatment area, provided the necessary capacities are available and the EOMC has been consulted.

\section{Conclusions}

When hospital resources are overwhelmed during the initial stage of a mass-casualty terrorist event, the provision of situation-adapted tactical and strategic casualty care in accordance with TDSC ${ }^{\circledR}$ principles, i.e. CATEGORISING, PRIORITISING, COORDINATING, and IMPLEMENTING, is an effective approach to managing a dynamic major incident.

An incident management approach that is tailored to the situation and based on tactical aspects provides the basis for medical care, the primary aim of which is to ensure the survival of as many patients as possible. This type of tactical medical care necessitates first and foremost the appropriate triage of the injured at the hospital.

Available resources, which are temporarily scarce in a mass-casualty terrorist incident, can then be deployed in a focused manner. This type of tactical and strategic management of patients in environments with strained resources can be effective also in conventional masscasualty incidents.

After categorisation, T1 (RED) patients have a primary survey. The purpose of this approach is to identify haemodynamically unstable patients with bleeding into body cavities as early as possible. These patients are prioritised for emergency surgical procedures and the necessary arrangements are made. Diagnostic procedures and additional measures are performed in order to ensure the success of surgery without causing a delay.

When resources are scarce during the initial stage of a mass-casualty terrorist event, TASC teams that have the skills needed to perform a primary survey are required. Fluid therapy should be restrictive (permissive hypotension) unless contraindicated. Clotting products are replaced in a standardised manner on the basis of patient requirements, which are calculated using rapidly available surrogates (blood gas analysis).

Blood products can be administered or kept available depending on risks and triage categories. The highest priority should be given to casualties who require immediate surgery for injuries associated with bleeding into body cavities $(\mathrm{T} 1++)$.

The recommendations and approaches described here should be considered as proposals for hospitals to develop standards or modify well-established standards that enable them to prepare themselves successfully for situations (e.g. mass-casualty terrorist or shooter incidents) in which their resources are temporarily overwhelmed.

In conclusion the TDSC course with its idea of a focussed in-hospital diagnostic work up and treatment, related to a staged process of initial categorization, prioritising, coordinating and implementing is directly connected to the well-established MRMI (Medical Response to Major Incidents \& Desasters) course. Herein a region or country is faced to one or more major incidents over a longer real time period and multiple causalities.

The MRMI course trains the mutual interaction of regionally available medical units for the management of a mass casualty incident both prehospital and in-hospital, taking into account different care levels.[46, 47] But the impact of in-hospital decision making especially related to different strategies of surgical treatment (TASC versus DCS) and/or the need of resources is not that simulated in detail. Both courses with their different aims and didactics complement one another and will improve treatment and care in a mass casualty situation.

\section{Compliance with ethical standards}

Conflict of interest Achatz $\mathrm{G}$ and Bieler $\mathrm{D}$ are active as instructors of the TDSC - Course. Friemert B and Franke A are active as directors and instructors of the TDSC-Course. Achatz G, Bieler D, Franke A and Friemert B developed the TDSC $\AA$-Course concept. Hoth P and Pape HC confirms that there is no conlict of interest.

\section{References}

1. Wurmb T, Kowalzik B, Rebuck J, Franke A, Cwojdzinski D, Bernstein N, et al. Bewältigung von besonderen Bedrohungslagen. Notfall + Rettungsmedizin. 2018;21(8):664-72.

2. Wurmb T, Friemert B. Die Rolle des Krankenhauses bei Bedrohungslagen. Notfall + Rettungsmedizin. 2018;21(7):585-9.

3. Hossfeld B, Wurmb T, Josse F, Helm M. Massenanfall von Verletzten-Besonderheiten von „bedrohlichen Lagen“. AINS. 2017;52(09):618-29.

4. Friemert B, Franke A, Schwab R, Hinck D, Achatz G, AG EKTC der DGU. Chirurgische Versorgungsstrategien beim TerrorMANV. Notfall + Rettungsmedizin. 2018;21(4):278-288

5. Bieler D, Kollig E, Achatz G, Friemert B, Franke A. Typische Verletzungen durch terrorassoziierte Ereignisse und ihre Implikationen für die Erstversorgung. Trauma und Berufskrankheit. 2018;20(3):177-87.

6. Güsgen C, Franke A, Hentsch S, Kollig E, Schwab R. Das Terroranschlagtrauma - eine eigene Entität des Polytraumas. Der Chirurg. 2017;88(10):821-9.

7. Franke A, Bieler D, Friemert B, Schwab R, Kollig E, Güsgen C. The first aid and hospital treatment of gunshot and blast injuries. Dtsch Arztebl Int. 2017;114(14):237-43.

8. Achatz G, Bieler D, Franke A, Friemert B. Terrorassoziierter Massenanfall von Verletzten (TerrorMANV). Trauma und Berufskrankheit. 2018;20(3):188-95.

9. Juncken K, Heller AR, Cwojdzinski D, Disch AC, Kleber C. Verteilung der Sichtungskategorien bei Terroranschlägen 
mit einem Massenanfall von Verletzten. Der Unfallchirurg. 2019;122(4):299-308.

10. Hauer T, Huschitt N, Klein F, Poloczek S, Albers P, Cwojdzinski $\mathrm{D}$, et al. Patientenversorgung bei Terroranschlägen. Notfall + Rettungsmedizin. 2018;21(4):267-77.

11. Henke TJ, Freund F, Wieprich D, Helm M, Bergold MN, Byhahn C. Der Terroranschlag von Berlin-Die Vorgeschichte, der Einsatz und die Konsequenzen aus präklinischer Sicht. Notarzt. 2017;33(02):54-60.

12. Franke A, Bieler D, Friemert B, Kollig E, Flohe S. Prä- und innerklinisches Management bei MANV und Terroranschlag. Der Chirurg. 2017;88(10):830-40.

13. Franke A, Bieler D, Friemert B, Kollig E, Flohe S. Preclinical and intrahospital management of mass casualties and terrorist incidents. Der Chirurg; Zeitschrift fur alle Gebiete der operativen Medizen. 2017;88(10):830-40.

14. Ashkenazi I, Turegano-Fuentes F, Einav S, Kessel B, Alfici R, Olsha O. Pitfalls to avoid in the medical management of mass casualty incidents following terrorist bombings: the hospital perspective. Eur J Trauma Emerg Surg. 2014;40(4):445-50.

15. Güsgen C, Schwab R, Kleber C. Sichtung und chirurgische Notfallversorgung im Katastrophenfall. Zentralbl Chir. 2018;143(02):121-30

16. Kleber C, Solarek A, Cwojdzinski D, Strehl M, Wrede C, Heinz $\mathrm{J}$, et al. Der Berliner Krankenhaus-Sichtungsalgorithmus für den Massenanfall von Verletzten. Der Unfallchirurg. 2019.

17. Bouillon B, Marzi I. The updated German "Polytrauma Guideline": an extensive literature evaluation and treatment recommendation for the care of the critically injured patient. Eur J Trauma Emerg Surg. 2018;44(Suppl 1):1.

18. Leenen M, Scholz A, Lefering R, Flohe S, TraumaRegister DGU. Limited volume resuscitation in hypotensive elderly multiple trauma is safe and prevents early clinical dilutive coagulopathy-a matched-pair analysis from TraumaRegister DGU((R)). Injury. 2014;45(Suppl 3):S59-63.

19. Schmidt BM, Rezende-Neto JB, Andrade MV, Winter PC, Carvalho MG Jr, Lisboa TA, et al. Permissive hypotension does not reduce regional organ perfusion compared to normotensive resuscitation: animal study with fluorescent microspheres. WJES. 2012;7(Suppl 1):S9.

20. Albreiki M, Voegeli D. Permissive hypotensive resuscitation in adult patients with traumatic haemorrhagic shock: a systematic review. Eur J Trauma Emerg Surg. 2018;44(2):191-202.

21. Carrick MM, Leonard J, Slone DS, Mains CW, Bar-Or D. Hypotensive resuscitation among trauma patients. Biomed Res Int. 2016;2016:8901938.

22. Mueller MM, Van Remoortel H, Meybohm P, Aranko K, Aubron C, Burger R, et al. Patient blood management: recommendations from the 2018 Frankfurt consensus conference. JAMA. 2019;321(10):983-97.

23. Frohlich M, Driessen A, Bohmer A, Nienaber U, Igressa A, Probst $\mathrm{C}$, et al. Is the shock index based classification of hypovolemic shock applicable in multiple injured patients with severe traumatic brain injury?-an analysis of the TraumaRegister DGU((R)). Scandinavian J Trauma Resusc Emerg Med. 2016;24(1):148.

24. Wafaisade A, Wyen H, Mutschler M, Lendemans S, Bouillon B, Flohe $\mathrm{S}$, et al. Current practice in coagulation and transfusion therapy in multiple trauma patients: a German nation-wide online survey. Der Unfallchirurg. 2015;118(12):1033-40.

25. Mutschler M, Nienaber U, Wafaisade A, Brockamp T, Probst C, Paffrath $\mathrm{T}$, et al. The impact of severe traumatic brain injury on a novel base deficit- based classification of hypovolemic shock. Scandinavian J Trauma Resusc Emerg Med. 2014;22:28.

26. Spahn DR, Bouillon B, Cerny V, Duranteau J, Filipescu D, Hunt $\mathrm{BJ}$, et al. The European guideline on management of major bleeding and coagulopathy following trauma: fifth edition. Critic care. 2019;23(1):98.

27. Hilbert P, Hofmann GO, Lefering R, Struck MF. Trauma bay haemoglobin level. Predictor of coagulation disorder in major trauma. Der Unfallchirurg. 2015;118(7):601-6.

28. Hilbert P, Hofmann GO, Teichmann J, Struck MF, Stuttmann R. The, "coagulation box" and a new hemoglobin-driven algorithm for bleeding control in patients with severe multiple traumas. Arch Trauma Res. 2013;2(1):3-10.

29. Hilbert P, Hofmann GO, zur Nieden K, Teichmann J, Jakubetz J, Stuttmann R. Coagulation management of trauma patients with unstabile circulation: establishment of a hemoglobinoriented standard operating procedure. Der Anaesthesist. 2012;61(8):703-10.

30. Keene DD, Nordmann GR, Woolley T. Rotational thromboelastometry-guided trauma resuscitation. Current opinion in critical care. 2013;19(6):605-12.

31. Schochl H, Schlimp CJ, Voelckel W. Perioperative coagulation management in multiple trauma patients based on viscoelastic test results. Der Unfallchirurg. 2014;117(2):111-7.

32. Spahn DR. TEG(R)- or ROTEM(R)-based individualized goaldirected coagulation algorithms: don't wait--act now! Critic Care. 2014;18(6):637.

33. Stein P, Kaserer A, Sprengel K, Wanner GA, Seifert B, Theusinger $\mathrm{OM}$, et al. Change of transfusion and treatment paradigm in major trauma patients. Anaesthesia. 2017;72(11):1317-26.

34. Theusinger OM, Stein P, Spahn DR. Transfusion strategy in multiple trauma patients. Curr Opin Critic Care. 2014;20(6):646-55.

35. Akoglu H, Celik OF, Celik A, Ergelen R, Onur O, Denizbasi A. Diagnostic accuracy of the Extended Focused Abdominal Sonography for Trauma (E-FAST) performed by emergency physicians compared to CT. Am J Emerg Med. 2018;36(6):1014-7.

36. Samuel AE, Chakrapani A, Moideen F. Accuracy of Extended Focused Assessment with Sonography in Trauma (e-FAST) performed by emergency medicine residents in a level one tertiary center of India. Adv J Emerg Med. 2018;2(2):e15.

37. Zanobetti M, Coppa A, Nazerian P, Grifoni S, Scorpiniti M, Innocenti F, et al. Chest Abdominal-Focused Assessment Sonography for Trauma during the primary survey in the emergency department: the CA-FAST protocol. Eur J Trauma Emerg Surg. 2018;44(6):805-10.

38. Huber-Wagner S, Biberthaler P, Haberle S, Wierer M, Dobritz $\mathrm{M}$, Rummeny E, et al. Whole-body CT in haemodynamically unstable severely injured patients - a retrospective, multicentre study. PLoS ONE. 2013;8(7):e68880.

39. Huber-Wagner S, Kanz KG, Hanschen $M$, van Griensven M, Biberthaler P, Lefering R. Whole-body computed tomography in severely injured patients. Curr Opin Critic Care. 2018;24(1):55-61.

40. Waldeck S, Franke A, Gusgen C, Schwab R, Kalff J, Pantelis D. SMAR(3)T(c)-a new time-saving diagnostic emergency room management algorithm. Zeitschrift fur alle Gebiete der operativen Medizen: Der Chirurg; 2019.

41. Bieler D, Horster A, Lefering R, Franke A, Waydhas C, HuberWagner $S$, et al. Evaluation of new quality indicators for the TraumaRegister DGU((R)) using the systematic QUALIFY methodology. European journal of trauma and emergency surgery : official publication of the European Trauma Society. 2018.

42. Bieler D, Franke AF, Hentsch S, Paffrath T, Willms A, Lefering $\mathrm{R}$, et al. Gunshot and stab wounds in Germany-epidemiology and outcome: analysis from the TraumaRegister DGU(R). Der Unfallchirurg. 2014;117(11):995-1004. 
43. Geissler RG, Kosters C, Franz D, Buddendick H, Borowski M, Juhra C, et al. Utilisation of Blood Components in Trauma Surgery: A Single-Centre, Retrospective Analysis before and after the Implementation of an Educative PBM Initiative. Transf Med Hemother. 2015;42(2):83-9.

44. Bala M, Kaufman T, Keidar A, Zelig O, Zamir G, Mudhi-Orenshat $\mathrm{S}$, et al. Defining the need for blood and blood products transfusion following suicide bombing attacks on a civilian population: a level I single-centre experience. Injury. 2014;45(1):50-5.

45. Noel S, Francois A, LeFailler F, Charpentier F, Baudonnet T, Bierling P, et al. Lessons learned from Paris and Nice. ISBT Sci Ser. 2018;13:35-46.
46. Lennquist Montan K, Hreckovski B, Dobson B, Ortenwall P, Montan C, Khorram-Manesh A, et al. Development and evaluation of a new simulation model for interactive training of the medical response to major incidents and disasters. Eur J Trauma Emerg Surg. 2014;40(4):429-43.

47. Montan KL, Ortenwall P, Lennquist S. Assessment of the accuracy of the Medical Response to Major Incidents (MRMI) course for interactive training of the response to major incidents and disasters. Am J Disaster Med. 2015;10(2):93-107. 